\title{
Flash nanoprecipitation for the production of endosomolytic polymersomes for cytosolic drug delivery
}

Hayden M. Pagendarm ${ }^{1 \wedge}$, Payton T. Stone ${ }^{2, \wedge}$, Jessalyn J. Baljon ${ }^{1}$, Mina H. Azizz,4, Lucinda E. Pastora ${ }^{2}$, John T. Wilson ${ }^{1,2,5-9^{*}}$

${ }^{1}$ Department of Biomedical Engineering, Vanderbilt University, Nashville, TN 37235, USA

${ }^{2}$ Department of Chemical and Biomolecular Engineering, Vanderbilt University, Nashville, TN 37235, USA

${ }^{3}$ Department of Biochemistry, Vanderbilt University, Nashville, TN 37235, USA

${ }^{4}$ Department of Neuroscience, Vanderbilt University, Nashville, TN 37235, USA

${ }^{5}$ Vanderbilt Institute for Infection, Immunology, and Inflammation, Vanderbilt University Medical Center, Nashville, TN 37232, USA

${ }^{6}$ Vanderbilt Center for Immunobiology, Vanderbilt University Medical Center, Nashville, TN 37232 , USA

${ }^{7}$ Vanderbilt Institute of Chemical Biology, Vanderbilt University, Nashville, TN, 37232

${ }^{8}$ Vanderbilt Institute of Nanoscale Science and Engineering, Vanderbilt University, Nashville, TN 37232

${ }^{9}$ Vanderbilt-Ingram Cancer Center, Vanderbilt University Medical Center, Nashville, TN 37232 , USA

$\wedge^{\wedge}$ These authors contributed equally to this work

${ }^{*}$ Corresponding Author: john.t.wilson@vanderbilt.edu 


\section{Abstract:}

The delivery of biomacromolecular drugs to cytosolic targets has been a long-standing engineering challenge due to the presence of multiple biological barriers including cellular and endosomal membranes. Although many promising carriers designed to facilitate endosomal escape have been developed, the clinical translation of these carriers is often limited by complex production processes that are not amenable to scaled-up manufacturing. In this study, we employed flash nanoprecipitation (FNP) for the rapid, scalable, and reproducible assembly of nanocarriers composed of the $\mathrm{pH}$-responsive, endosomolytic diblock copolymer poly[(ethylene glycol) $x_{x}$-block-[((2-diethylamino) ethyl methacrylate) $)_{0.6}$-co-(butyl methacrylate) $)_{0.4]_{y}}$ (PEG-bDEAEMA-Co-BMA). We found that varying the second block molecular weight, while holding the first block molecular weight constant, significantly influenced nanoparticle self-assembly and hence nanocarrier properties and function - including drug encapsulation, endosomolytic capacity, cytotoxicity, and in vitro activity of a cytosolically-active drug cargo, a cyclic dinucleotide (CDN) stimulator of interferon genes (STING) agonist. We found that while increasing second block molecular weight enhanced the capacity of nanocarriers to induce endosomal destabilization, larger second block molecular weights also lead to increased cytotoxicity, increased particle size and heterogeneity, increased the encapsulation efficiency of small $(<0.5$ $\mathrm{kDa})$ hydrophilic drugs, decreased the encapsulation efficiency of large (10 kDa) hydrophilic biomacromolecules, and decreased long-term particle stability. Collectively, these results demonstrate the utility of FNP for the rapid and scalable production of uniform PEG- $b$-DEAEMAco-BMA nanocarriers and implicate an optimal hydrophilic mass fraction for balancing desirable nanoparticle properties with cytosolic cargo delivery efficiency.

\section{Introduction}

Many classes of therapeutic biomacromolecules, including siRNA, miRNA, mRNA, peptides, and proteins, act on intracellular targets to induce a desired cellular and/or physiological response. Unfortunately, these biomacromolecular therapeutics typically suffer from low 
membrane permeability and thus rarely reach their cytosolic targets with sufficient efficiency to exert therapeutic effects. ${ }^{1}$ This challenge has led to the development of a variety of cytosolic delivery systems, including polymeric drug carriers, that serve to facilitate the endosomal escape and cytosolic release of their associated cargo. ${ }^{2-4}$ These systems generally rely upon protonatable species to induce destabilization of the endosomal membrane as endosomal $\mathrm{pH}$ drops during endolysosomal trafficking. By absorbing protons, these species develop a netpositive charge, stimulating interaction with the negatively charged endosomal membrane. This interaction has been exploited to introduce hydrophobic moieties, capable of mediating endosomal membrane disruption, to the endosomal membrane through the copolymerization of protonatable amino monomers $\left(\mathrm{pK}_{\mathrm{a}} \sim 6.2-7\right)$ with hydrophobic monomers capable of mediating endosomal disruption. ${ }^{5-10}$

Previously, our laboratory has described several classes of STING-activating nanoparticles (STANs). ${ }^{11-13}$ STANs are composed of the pH-responsive, endosomolytic diblock copolymer poly[(ethylene glycol $)_{x}-$ block-[((2-diethylamino) ethyl methacrylate $)_{0.6}$-co-(butyl methacrylate) 0.4 $_{\text {y }}$ (PEG-b-DEAEMA-co-BMA), and exploit the aforementioned mechanism to induce disruption of the endosomal membrane for the cytosolic delivery of cyclic dinucleotides (CDNs), which are agonists of the stimulator of interferon genes (STING) pathway. ${ }^{11,12}$ The STING pathway is triggered when the enzyme cyclic-GMP-AMP synthase (cGAS) detects DNA in the cytoplasm, a common indicator of viral infection. ${ }^{14-17}$ cGAS subsequently synthesizes $2{ }^{\prime}, 5-3^{\prime} 5^{\prime}$ cyclic guanosine monophosphate-adenosine monophosphate (cGAMP), the endogenous ligand for STING. ${ }^{18,19}$ Activation of STING triggers a type I interferon (IFN-I)-driven inflammatory response that stimulates dendritic cells to cross-present antigen on class I major histocompatibility complex (MHC). This pathway can be exploited to cross-present tumor antigens in order to prime antitumor $\mathrm{CD}^{+}$cytotoxic T cells. ${ }^{20}$

As cGAMP is an anionic, hydrophilic molecule, it does not readily cross the cellular plasma membrane, is poorly endocytosed, and thus rarely reaches the cytosol where STING is 
located. ${ }^{21,22}$ We have previously demonstrated that STANs enhance the cytosolic delivery of cGAMP, causing robust antitumor $\mathrm{T}$ cell activation and subsequent tumor elimination and prevention or tumor rechallenge in multiple models of murine cancers. ${ }^{11,23}$ In our previous studies, we utilized a previously-described direct hydration approach for STAN production, ${ }^{24}$ which enabled the self-assembly of polymeric vesicles, or polymersomes, with an aqueous core for cGAMP loading and a pH-responsive shell to induce endosomal membrane destabilization. ${ }^{11,12}$ This approach involved the dropwise addition of solubilized cGAMP to a highly concentrated solution of swollen polymer in ethanol $(\mathrm{EtOH})$. Although this direct hydration method allowed for high encapsulation efficiency (EE) of cGAMP, the process is conducted on the scale of a few hundred microliters and accordingly cannot be implemented into a practical scaled-up manufacturing process. Additionally, batches of STANs produced via direct hydration could be quite heterogeneous in size, with a typical PDI of $\sim 0.2-0.3$ as reported by DLS, and often contained non-vesicular structures of micellar or filamentous morphologies. ${ }^{13}$ Furthermore, polymersome assembly was only favored using a relatively short $6 \mathrm{kDa}$ second block, which alone weakly disrupted cell membranes in vitro, necessitating copolymerization of thiol-reactive pyridyl disulfide methacrylate (PDSMA) groups into the second block of the polymer to allow for postassembly crosslinking of the polymersomes, effectively resulting in an increase in the molecular weight of the polymer chains. Although this increase in molecular weight resulted in an improvement in membrane destabilization, the crosslinking process is complex and requires additional steps, further limiting the scalability of direct hydration.

This study focused on optimizing a flash nanoprecipitation (FNP) process for the scalable and reproducible production of homogenously sized STANs. Specifically, an organic solution containing PEG- $b$-DEAEMA-co-BMA was simultaneously impinged against an aqueous solution containing hydrophilic cargo in a confined impingement jet (CIJ) mixer (Fig. 1A). The turbulent mixing conditions within the confined impinging jet (ClJ) enable the spontaneous self-assembly of nanoparticles and simultaneous encapsulation of both hydrophilic and hydrophobic cargo within 
milliseconds. ${ }^{25-27}$ Previous studies using other diblock copolymers reported that FNP facilitated the generation of polymersomes with many advantageous properties including $<100 \mathrm{~nm}$ particle size, low PDI, and high EE. ${ }^{26,28,29}$ Herein, we report that using FNP with second block molecular weights ranging from $6 \mathrm{kDa}$ to $20 \mathrm{kDa}$ enabled the formulation of stable and cytosolically active STANs of smaller size $(<100 \mathrm{~nm})$, with a low PDI $(<0.1)$, a polymersome morphology, and without the need for post-processing crosslinking. We demonstrated that although increasing second block molecular weight enhanced the capacity of nanocarriers to induce endosomal destabilization, larger second block molecular weights also lead to increased cytotoxicity, increased particle size and heterogeneity, increased the encapsulation efficiency of small $(<0.5$ kDa) hydrophilic drugs, decreased the encapsulation efficiency of large (10 kDa) hydrophilic biomacromolecules, and decreased long-term particle stability. Collectively, these results implicate that there is a balance between second block molecular weight, nanoparticle properties, and capacity for endosomal disruption that can be optimized to tune carriers to deliver diverse categories of biomacromolecular drug cargo to achieve a wide-range of therapeutic applications.

\section{Materials and Methods}

\subsection{Materials}

All materials were purchased from Sigma-Aldrich unless otherwise specified.

\subsection{Methods}

\subsubsection{Polymer Synthesis}

The reversible addition-fragmentation chain transfer (RAFT) polymer synthesis method utilized in this experiment has been described previously. ${ }^{11}$ Briefly, the monomers, butyl methacrylate (BMA) and 2-(diethylamino) ethyl methacrylate (DEAEMA) (Tokyo Chemical Industry) were reacted with poly(ethylene glycol) 4-cyano-4-(phenylcarbonothioylthio)pentanoate (PEG CTA) and 4-4'-AZO-bis(4-cyanovaleric acid) initiator (V501) (MP Biomedicals) for $18 \mathrm{~h}$ at $70^{\circ} \mathrm{C}$ in a reaction vessel following a 30 min purge with gaseous nitrogen. Polymers were then purified via dialysis against acetone, followed by a $50 \%$ acetone $50 \%$ de-ionized water (DI $\mathrm{H}_{2} \mathrm{O}$ ) 
mixture, followed by $100 \%$ DI $\mathrm{H}_{2} \mathrm{O}$ for a total of $48 \mathrm{~h}$ in 3500 Da MWCO SnakeSkin dialysis tubing (Thermo Scientific) and subsequently lyophilized for $48 \mathrm{~h}$ in a FreeZone Benchtop Freeze Dry System (Labconco). ${ }^{1} \mathrm{H}-\mathrm{NMR}$ spectroscopy in $\mathrm{CdCl}_{3}$ was analyzed pre- and post-reaction and after lyophilization using a $400 \mathrm{MHz}$ NMR Spectrometer (Bruker), courtesy of Vanderbilt University's Small Molecule NMR Facility Core, to determine conversion and composition, respectively.

\subsubsection{Nanoparticle fabrication via flash nanoprecipitation}

Nanoparticles were formulated using a multi-stream combined impingement jet (CIJ) (Holland Applied Technologies). For each sample, $10 \mathrm{mg}$ of polymer was dissolved in $1 \mathrm{~mL}$ of tetrahydrofuran (THF) and then aspirated into a disposable $1 \mathrm{~mL}$ polypropylene syringe. For experiments loading hydrophobic drug cargo, the hydrophobic drug was also dissolved at the indicated concentration in the THF syringe containing the polymer solution. A second syringe was used to aspirate $1 \mathrm{~mL}$ of an aqueous solution containing either $\mathrm{DI}_{2} \mathrm{O}$ alone for empty nanoparticles or solubilized hydrophilic drug. The syringes were then attached at the inlets of the CIJ unit, and both were pressed down simultaneously with constant force at high speed to induce turbulent mixing within the chamber. For each impingement, the resulting mixture was collected in a $20 \mathrm{~mL}$ scintillation vial, and the total volume was split evenly between the two syringes. The impingement process was repeated four times for a total of five impingements per batch of fabricated nanoparticles. On the final impingement, the mixture was collected in a quench bath of $4 \mathrm{~mL}$ of $\mathrm{DI} \mathrm{H}_{2} \mathrm{O}$ under vigorous stirring in order to induce chain stabilization and establish the vesicular morphology of the particles. The holdup volume - the liquid occupying the piping within the $\mathrm{ClJ}$ - of approximately $0.25 \mathrm{~mL}$ was not permitted to enter the quench bath as it did not undergo turbulent mixing.

\subsubsection{Size, PDI, and Zeta Potential Measurements}

Size, PDI, and zeta potential values of nanoparticle samples were analyzed using a Zetasizer Nano ZS (Malvern Panalytical). For size and PDI, purified sample was diluted 10x in 
$0.22 \mu \mathrm{m}$ sterile-filtered phosphate-buffered saline (PBS) (Gibco) in a $1.5 \mathrm{~mL}$ semi-micro cuvette and analyzed using the ZS. For zeta potential, purified sample was diluted 10x in $0.22 \mu \mathrm{m}$ sterilefiltered $11.1 \mathrm{mM} \mathrm{NaCl}$ (for a final concentration of $10 \mathrm{mM} \mathrm{NaCl}$ ), and transferred to a DTS1070 capillary cell for measurement.

\subsubsection{Encapsulation Efficiency Quantification}

After formulation, drug-loaded samples were placed in a $1 \mathrm{~L}$ beaker of $\mathrm{DI} \mathrm{H}_{2} \mathrm{O}$ and purified via dialysis against $\mathrm{DI} \mathrm{H}_{2} \mathrm{O}$ using $3.5 \mathrm{kDa}$ MWCO snakeskin dialysis tubing for $48 \mathrm{~h}$ to remove any unencapsulated drug and residual THF from the formulation process. $\mathrm{DI}_{2} \mathrm{O}$ was exchanged approximately every $8 \mathrm{~h}$ during this procedure. Nile red loaded samples were further purified via gravimetric filtration using qualitative filter paper (Whatman), as unencapsulated Nile red precipitated in aqueous solution. Purified samples were diluted $2 \mathrm{x}$ in $\mathrm{EtOH}$ to disrupt the polymersome architecture and release encapsulated drug, diluted to a measurable concentration, and analyzed for fluorescence intensity using a Synergy-H1 plate reader (BioTek). Fluorescent readouts were normalized to a blank, and model drug concentrations were determined by use of a standard curve of known concentrations. Encapsulation efficiency (EE) was calculated as the ratio of the mass of drug encapsulated in the purified sample to the total mass of drug added into the system. Loading capacity (LC) was calculated as the ratio of the mass of drug encapsulated in the purified sample to the mass of the full nanoparticle formulation.

\subsubsection{Transmission Electron Microscope (TEM) Imaging of Nanoparticles}

To examine the morphology of the nanoparticles, a $200 \mathrm{kV}$ Osiris Transmission Electron Microscope was used, courtesy of the Vanderbilt Institute of Nanoscale Science and Engineering (VINSE). For each sample, $10 \mu \mathrm{L}$ of a $1 \mathrm{mg} / \mathrm{mL}$ nanoparticle suspension was drop-casted onto commercial Carbon Type B TEM grids (Ted Pella, Inc.), dried, and stained with $1 \%$ methylamine tungstate for imaging (Molecular Probes).

\subsubsection{Hemolysis Assay}


A Genesys-150 ultraviolet-visible (UV/vis) spectrophotometer (Thermo Scientific) was used to determine exact polymer concentrations of nanoparticle formulations after dialysis, by comparison of the absorbance of the sample at $310 \mathrm{~nm}$ to that of standards of known polymer concentrations. Nanoparticles were then diluted to concentrations of 10,5 , and $1 \mu \mathrm{g} / \mathrm{mL} .7 \mu \mathrm{L}$ of each formulation was placed into a 96 -well V-bottom plate (Greiner Bio-One), and $7 \mu \mathrm{L}$ of $0.1 \%$ Triton X-100 and 1x PBS were used as positive and negative controls, respectively. De-identified human whole blood, obtained courtesy of the Vanderbilt Hematology Core, was washed by centrifuging at $450 \times \mathrm{g}$ for $5 \mathrm{~min}$, aspirating off the plasma layer, resuspending the hematocrit in 1x PBS, and repeating the washing procedure four times. The resulting hematocrit was then diluted 50x in PBS at varying $\mathrm{pH}$ associated with endolysosomal trafficking $(\mathrm{pH}$ 5.8, 6.2, 6.6, 7.0, and 7.4). $168 \mu \mathrm{L}$ of diluted blood was added to each nanoparticle formulation in the 96-well Vbottom plate, and suspensions were incubated at $37^{\circ} \mathrm{C}$ for $1 \mathrm{~h}$. Plates were then centrifuged at $600 \times \mathrm{g}$ for $1 \mathrm{~min}$, and $80 \mu \mathrm{L}$ of supernatant was collected and transferred to a clear, 96-well flat bottom plate (Greiner Bio-One). The amount of hemoglobin leakage into the supernatant was determined via absorbance spectroscopy (Synergy $\mathrm{H} 1, \lambda=575 \mathrm{~nm}$ ), and percent hemolysis was calculated as the ratio of (Abssample $\left.-\mathrm{AbS} \mathrm{PBS}_{\mathrm{P}}\right) /\left(\mathrm{AbS}_{\text {Triton }}-\mathrm{AbS} \mathrm{SBS}_{\mathrm{PB}}\right)$.

\subsubsection{Gal8-YFP Assay}

Following the use of UV/Vis spectroscopy to determine the nanoparticle concentration following dialysis, nanoparticles were concentrated using 3 kDa Amicon® Ultra-15 Centrifugal Filter Units (Millipore) by 45 min of centrifugation at $4600 \mathrm{xg}$ and subsequent resuspension in $1 \mathrm{x}$ PBS to a final concentration of $5 \mathrm{mg} / \mathrm{mL}$. Gal8 recruitment assays were then performed as previously described ${ }^{30}$ with minor modifications as follows. MDA-MB-231 cells stably expressing Gal8-YFP were seeded in 96-well black walled clear bottom plates (Grenier, catalog number 655090) at a density of 5000 cells/well and allowed to adhere overnight. The following day, cells were treated with nanoparticle formulations at indicated concentrations and incubated for an additional 15 hours. Media was then exchanged with $100 \mu \mathrm{L}$ of imaging media (FluoroBrite DMEM 
supplemented with 25 mM HEPES, 10\% FBS, 1\% Pen/Strep, and $4 \mu \mathrm{M}$ Hoechst 33342). Cells were imaged using an ImageXpress Nano Automated Imaging System (Molecular Devices) with a 20 x Nikon CFI60 series objective, courtesy of the Vanderbilt High Throughput Screening Core. Images were analyzed using the Transfluor Application Module within the MetaXpress Software (Molecular Devices), which blindly counted the pixel area of the Gal8+ spots within each image.

\subsubsection{Cell Culture}

A549 human adenocarcinoma cells (Invivogen) were maintained in Dulbecco's modified Eagle's medium (Gibco) supplemented with 10\% fetal bovine serum (Invitrogen, Carlsbad, CA), $100 \mathrm{U} / \mathrm{mL}$ penicillin (Gibco), $100 \mu \mathrm{g} / \mathrm{mL}$ streptomycin (Gibco). MDA-MB-231 epithelial human breast cancer cells engineered to express a Galectin 8 (Gal8) Yellow Fluorescent Protein (YFP) fusion (MDA-231-YFP) were obtained courtesy of the developers of a previously described Gal8 recruitment assay. ${ }^{30}$ MDA-231-YFP cells were maintained in Dulbecco's modified Eagle's medium (Gibco) supplemented with $10 \%$ fetal bovine serum (Invitrogen, Carlsbad, CA), $100 \mathrm{U} / \mathrm{mL}$ penicillin (Gibco), and $100 \mu \mathrm{g} / \mathrm{mL}$ streptomycin (Gibco). THP1-Dual monocytes (Invivogen) with an IFN regulatory factor (IRF)-inducible reporter construct were cultured in RPMI (Gibco) supplemented with $2 \mathrm{mM}$ L-glutamine, 25mM HEPES, $10 \%$ FBS, $1 \%$ penicillin and streptomycin, and $100 \mu \mathrm{g} / \mathrm{mL}$ Normocin. $100 \mu \mathrm{g} / \mathrm{mL}$ zeocin and $10 \mu \mathrm{g} / \mathrm{mL}$ blasticidin was added every other passage to maintain selection pressure. All cells were passaged when confluency reached 7090\% using 0.01\% Trypsin/EDTA (Gibco).

\subsubsection{Cell Viability}

Following the use of UV/Vis spectroscopy to determine the nanoparticle concentration following dialysis, nanoparticles were concentrated using 3 kDa Amicon® Ultra-15 Centrifugal Filter Units (Millipore) by 45 min of centrifugation at $4600 \mathrm{xg}$ and subsequent resuspension in $1 \mathrm{x}$ PBS to a final concentration of $50 \mathrm{mg} / \mathrm{mL}$. Cell viability was measured using a CellTiter-Glo Luminescent Cell Viability Assay (Promega). Briefly, A549 human adenocarcinoma cells were seeded at a density of 5000 cells/well in a clear-bottom, white-walled 96-well plate (Greiner Bio- 
One) and treated with empty nanoparticle formulations at indicated concentrations for 24 hours. CellTiter-Glo reagent was added, and the plate was allowed to incubate for 30 mins before measuring luminescence using a BIO-TEK Synergy $\mathrm{HI}$ plate reader. Relative viability was calculated by normalizing luminescence readings to a control group of cells treated with PBS. IC $\mathrm{C}_{50}$ values were extrapolated by performing a nonlinear regression curve fit on the cytotoxicity data points using Prism 9.2.0 (GraphPad) software.

\subsubsection{Dose Response}

cGAMP was dissolved at $0.5 \mathrm{mg} / \mathrm{mL}$ in $1 \mathrm{~mL}$ of $\mathrm{DI} \mathrm{H}_{2} \mathrm{O}$ and impinged five times against $10 \mathrm{mg} / \mathrm{mL}$ of polymer in $1 \mathrm{~mL}$ of THF. cGAMP activity was measured using THP1-Dual cells (InvivoGen) expressing an IFN regulatory factor (IRF)-inducible reporter construct. Cells were seeded in a 96-well plate at a density of 20,000 cells/well, and then treated with indicated concentrations of nanocarrier formulations or free cGAMP for $24 \mathrm{~h}$. Cells were treated with QUANTI-Luc reagent (InvivoGen), and relative expression of interferon was measured via luminescence using a BIO-TEK Synergy $\mathrm{HI}$ plate reader.

\subsubsection{Statistical Analysis}

All statistical analysis was performed using GraphPad Prism version 9.2.0 software. Error bars on graphs denote standard deviation.

\section{Results and Discussion}

\section{$\underline{3.1 \text { Polymer library and process variables }}$}

\subsubsection{Polymer characterization}

Polymers were synthesized according to a procedure described in previous work. ${ }^{11}$ Briefly, RAFT polymerization was utilized to synthesize the poly(ethylene glycol) $)_{x}$-block-[(2diethylamino)ethyl methacrylate) ${ }_{0.6}$-co-(butyl methacrylate) $\left.)_{0.4}\right]_{y}$ copolymer. The PEG group forms a hydrophilic shell to provide colloidal stability in aqueous solution and enhance biocompatibility. Following endosomal acidification, the DEAEMA groups became protonated resulting in solubilization of the second block, disassembly of the nanoparticle, and exposure of the 
membrane-Iytic DEAEMA-co-BMA domains, resulting in interactions with and the disruption of the endosomal membrane due to electrostatic attraction, resulting in disruption of the endosomal membrane. ${ }^{12,31}$ A library of polymers was synthesized to examine the effect of second block molecular weight (MW) on the properties of the resultant nanoparticles while first block MW was held constant at $2 \mathrm{kDa}$, as it has been reported that polymer hydrophilic mass fraction affects nanoparticle self-assembly as well as endosomolytic capacity. Note: For the remainder of the paper, a $\mathrm{PEG}_{2 \mathrm{k}}-\mathrm{EB}_{\mathrm{x}^{\prime} \mathrm{k}}$ copolymer will refer to a copolymer with a [DEAEMA-co-BMA] (E-co-B) block molecular weight of ' $x$ ' kDa. Our polymer library consisted of four polymers: $P E_{2 k}-E_{6 k}$, $P E G_{2 k}-E_{12 k}, P E G_{2 k}-E_{15 k}$, and $P E G_{2 k}-E_{20 k}$. Polymers were characterized for conversion and composition using proton nuclear magnetic resonance $\left({ }^{1} \mathrm{H}-\mathrm{NMR}\right)$. Polymer properties are described in Table 1.

\subsubsection{Optimization of process variables}

We first examined the effect of organic solvent choice on nanocarrier structure and function. We primarily examined Hildebrand solubility parameters $(\delta)$, which provide an indication of whether two compounds will be miscible. First, ethanol $(\mathrm{EtOH})$ was used as the organic solvent based on its use in our previous work with the direct hydration method of nanoparticle synthesis, ${ }^{24}$ but DLS analysis indicated that this resulted in the production of particles with a high PDI and intensity-weighted size distributions outside of the range of most polymersomes, most likely a micellar morphology (Fig. 1A). We then chose to examine tetrahydrofuran (THF), as it has a Hildebrand solubility parameter of $18.6 \mathrm{MPa}^{1 / 2}$, less than $\mathrm{EtOH}\left(\delta=26.2 \mathrm{MPa}^{1 / 2}\right)$. Analyses confirmed that all nanocarriers fabricated with THF as the organic solvent were monodisperse with size distributions within the range of those reported for polymersomes (Fig. 1B). ${ }^{26,32}$

We next examined the effect of the number of impingements on the resulting nanoparticles, as previous work in a multi-impingement flash nanoprecipitation study found that both the number of impingements and hydrophilic mass fraction significantly affected particle self- 
assembly and morphology. Manipulation of these parameters produced several morphologies including polymersomes, micelles, fibromicelles, and multi-compartmental vesicles. ${ }^{26}$ Polymersomes have displayed remarkable potential for drug delivery applications due to their improved physical and chemical stability compared to other morphologies. ${ }^{33,34}$ In addition, some polymersomes have been found to offer greater mechanical strength and enhanced serum stability than liposomes. ${ }^{35-37}$ Because of this, we sought to obtain a polymersomal morphology for our lead nanocarrier candidates.

It was determined that one impingement of all four diblock copolymers produced nanocarrier samples with a relatively low PDI of $\sim 0.1-0.2$ (Fig. 2B, D) and a z-average diameter of $\sim 30 \mathrm{~nm}$ (Fig. 2C, D). A diameter of this size is smaller than polymersomes assembled via direct hydration and is more consistent with a micellar morphology, which has been reported to limit cGAMP loading and activity. ${ }^{11}$ Interestingly, increasing the number of impingements from one to five caused a reduction in PDI for the $P E G_{2 k}-E B_{6 k}$ and $P E G_{2 k}-E B_{12 k}$ nanoparticles (Fig. 2B, E), and for all polymers, the particle size increased to a range of $\sim 80$ to $500 \mathrm{~nm}$ that is more consistent with a polymersome morphology (Fig. 2C, E). Increasing to ten impingements did not noticeably affect sample PDI or size (Fig. 2B, C, F), and therefore five impingements was selected for all subsequent studies.

\subsection{Effect of $2^{\text {nd }}$ block molecular weight on nanoparticle self-assembly}

\subsubsection{Characterization of physical properties}

Empty nanocarriers were formulated from each polymer via FNP with five impingements at a concentration of $10 \mathrm{mg} / \mathrm{mL}$ in the organic stream. Particle surface charge as measured via zeta potential exhibited an increasing trend with increasing second block molecular weight (Fig. 3A). This is potentially due to a reduction in PEG-shielding as second block molecular weight is increased, as the $2 \mathrm{kDa}$ PEG block may be unable to completely shield all of the cationic DEAEMA

groups of the polymers with larger second block molecular weights. ${ }^{38,39}$ We next sought to determine the effect of varying polymer second block molecular weight on the resultant 
nanoparticle polydispersity. If implemented into a large-scale manufacturing process, it is important that the batches of nanocarriers produced via FNP are uniform in size to avoid batchto-batch variability and meet GMP standards. As copolymer second block molecular weight was increased, both nanoparticle PDI and Z-average diameter increased as well (Fig. 3B, C). As one of the main goals of this study was to produce nanoparticles of relatively small size $(<100 \mathrm{~nm})$ and low polydispersity $(<0.1)$, this data indicates that all nanocarriers meet or very nearly meet the desired criteria, demonstrating the versatility of FNP to produce nanocarriers comprised of polymers with different second block sizes and hydrophilic mass fractions.

Nanoparticle morphology was evaluated via transmission electron microscopy (TEM). Representative TEM images of each polymeric nanocarrier are shown: $P E G_{2 k}-E_{6 k}$ (Fig. 4A), $\mathrm{PEG}_{2 \mathrm{k}}-\mathrm{EB}_{12 \mathrm{k}}$ (Fig. 4B), $\mathrm{PEG}_{2 \mathrm{k}}-\mathrm{EB}_{15 \mathrm{k}}$ (Fig. 4C), and $\mathrm{PEG}_{2 \mathrm{k}}-\mathrm{EB}_{20 \mathrm{k}}$ (Fig. 4D). TEM indicated that each nanocarrier exhibited a polymersome morphology as evidenced by the size, spherical structure, and well-defined boundaries of the particles.

Nanocarriers were also characterized for long-term colloidal stability. Specifically, nanocarriers were stored at room temperature and size (Fig. 5A), z-average diameter (Fig. 5B), and count rate (Fig. 5C), as reported by DLS, were tracked over a course of 15 weeks. Results indicate that nanocarriers with larger second block molecular weights sedimented from solution faster, as nanocarriers assembled using $P E G_{2 k}-E_{20 k}$ polymers sedimented within 1 week, $P E G_{2 k^{-}}$ $E_{12 k}$ nanocarriers settled out after 4 weeks, and $P E G_{2 k}-E_{6 k}$ nanocarriers remained stable for up to 15 weeks, as evident by the decreasing count rate (Fig. 5C) which is indicative of particle aggregation. ${ }^{40}$

\subsubsection{Drug encapsulation efficiency and loading capacity}

Next, we examined the loading capabilities of each of the nanocarriers. As previously reported in the literature, FNP has been found to encapsulate a variety of both hydrophilic and hydrophobic cargo within polymersomes. ${ }^{26}$ Model drugs were first used to give an accurate representation of the loading capabilities of the polymersomes. Sulforhodamine B (SRB) (0.5 kDa) 
and fluorescein isothiocyanate-Dextran (FITC-Dextran) (10 kDa) were utilized, as SRB is an anionic, hydrophilic dye (analogous to cGAMP) and FITC-Dextran is a neutral, hydrophilic macromolecule. SRB was generally encapsulated much more efficiently within the nanocarriers than FITC-Dextran and loaded at a much higher capacity than FITC-Dextran (Fig. 6A, B). This could be attributed to the lower molecular weight of SRB or electrostatic interactions between the negatively charged SRB molecules and the cationic DEAEMA groups of the polymer that may increase loading. The latter may also explain why SRB encapsulation efficiency generally increased as polymer second block molecular weight, and hence molar concentration of DEAEMA groups, increased (Fig. 6A). Nile Red (0.3 kDa) was used as a model drug to examine the loading of a hydrophobic molecule within each polymeric nanocarrier. Loading was significantly lower compared to hydrophilic cargo (Fig. 6C), indicating that the self-assembled nanomaterials may not be as suitable of a platform for the loading of hydrophobic molecules. Future analysis will examine the loading of other hydrophobic cargo to further confirm this.

3.3 Dependence of $2^{\text {nd }}$ block molecular weight on nanoparticle $\mathrm{pH}$-responsive properties

\subsubsection{Particle size change with decreasing $\mathrm{pH}$}

We next sought to examine the membrane destabilizing activity of the nanocarriers. Upon endocytosis and endo/lysosomal trafficking, the nanoparticles experience a decrease in environmental $\mathrm{pH}$ resulting in particle disassembly and subsequent release and endosomal escape of drug payloads. ${ }^{5-10}$ To assess pH-responsive nanoparticle disassembly, we measured size distributions of nanocarriers suspended in PBS buffers at physiological $\mathrm{pH}(7.4), \mathrm{pH} 6.6$, and pH 5.8 (the latter two are consistent with endosomal $\mathrm{pH}$ ). All four nanocarriers displayed a reduction in size as buffer $\mathrm{pH}$ decreased (Fig. 7A), indicating that all nanocarriers are responsive to $\mathrm{pH}$ decreases at levels relevant to endosomal acidification.

\subsubsection{Erythrocyte hemolysis}

To evaluate $\mathrm{pH}$-dependent membrane-destabilizing activity, a hemolysis assay was performed in which erythrocytes were incubated with each nanocarrier at different $\mathrm{pH}$ buffers and 
analyzed for hemoglobin release upon cell lysis. Trends for hemolysis activity were similar to $\mathrm{pH}$ responsive particle disassembly. Erythrocytes were lysed most effectively at endosomal pH for all four nanocarriers, with the $\mathrm{PEG}_{2 \mathrm{k}}-\mathrm{EB}_{20 \mathrm{k}}$ nanocarrier demonstrating the highest hemolysis (Fig. 7B). This was expected as the larger nanocarrier will have more $\mathrm{pH}$-responsive DEAEMA groups that can be protonated within the endosome and more exposed membrane-lytic domains to disrupt erythrocyte cell membranes. ${ }^{11,30}$ Minimal hemolysis was induced at physiological $\mathrm{pH}(7.4)$, indicating that the carriers maintain their structure when interacting with biological membranes outside of the endosome. Ultimately, these results demonstrate the ability of the nanocarriers to disassemble and disrupt biological membranes in response to a decrease in $\mathrm{pH}$, supporting their potential for enhancing cytosolic delivery of drug payloads.

\subsubsection{Galectin recruitment}

To further evaluate the capacity of nanocarriers to induce endosomal escape, we utilized a Gal8-YFP endosomal recruitment assay (Fig. 8A). Gal8-YFP is a fusion protein between Galectin 8 (Gal8), an endogenous cytosolically dispersed protein that binds carbohydrates, such as intralumenal endosomal glycans, and yellow fluorescent protein (YFP). This enables the qualitative and quantitative tracking of Gal8 redistribution from the cytosol to endosomes following endosomal membrane disruption. Increasing Gal8 recruitment directly correlates with increasing endosomolysis, and has been shown to correlate with increased bioactivity of cytosolic-acting biomacromolecular drugs. Here, we used a Gal8-YFP-expressing MDA-MB-231 breast cancer cell line that was previously validated to quantify the nanoparticle-mediated disruption of endosomal membranes following endosomal acidification. ${ }^{30}$ Representative images of MDA-231MB-YFP cells treated with $500 \mu \mathrm{g} / \mathrm{mL}$ of each nanocarrier are displayed (Fig. 8B). As expected, and consistent with the hemolysis data, endosomal disruption decreased with decreasing second block molecular weight and decreasing nanocarrier dose (Fig. 7C). Unexpectedly, we found that $P E G_{2 k}-E_{6 k}$ and $P E G_{2 k}-E_{12 k}$ exhibited minimal endosomolysis at all doses despite exhibiting 
hemolytic activity at pH 5.8 (Fig. 7C). Similar discrepancies have been previously reported suggesting that hemolytic activity is not entirely predictive of endosomal escape. ${ }^{30}$

3.4 Cytosolic drug delivery efficiency

\subsubsection{Effect of $2^{\text {nd }}$ block molecular weight on nanoparticle cytotoxicity}

We next evaluated the cytocompatibility of the fabricated nanocarriers by measuring carrier-induced cytotoxicity in A549 human adenocarcinoma cells using a CellTiter-Glo assay. Relative viability was plotted as a function of nanocarrier dose $(\mu \mathrm{M})$, and the half-maximal inhibitory concentration $\left(\mathrm{IC}_{50}\right)$ values were determined by a nonlinear regression curve fit (Fig. 9A). Our findings indicate that as nanocarrier molecular weight increases, the half-maximal inhibitory concentration of cell viability decreases (Fig. 9B). As induced cytotoxicity is a function of mass, it requires more moles of nanoparticles with a smaller molecular weight to induce the same toxicity on cells as nanoparticles with larger molecular weights (Fig. 9A).

\subsubsection{Delivery of cGAMP}

Due to the significant cytotoxicity observed with the $P E G_{2 k}-E B_{15 k}$ and $P E G_{2 k}-E B_{20 k}$ formulations (Fig. 9A), $P E G_{2 k}-E_{6 k}$ and $P E G_{2 k}-E_{12 k}$ carriers were chosen as lead candidates for further in vitro analysis. In particular, we sought to examine the ability of vehicles loaded with cyclic guanosine monophosphate-adenosine monophosphate (cGAMP) to induce STING pathway activation in THP1-Dual reporter monocytes. cGAMP, a cyclic dinucleotide (CDN) and the endogenous ligand of the stimulator of interferon genes (STING) pathway, is a promising therapeutic in cancer immunotherapeutic applications. However, CDNs have poor cell membrane permeability and therefore access the cytosol where STING is localized with low efficiency. We have previously demonstrated that polymersomes assembled using PEG- $b$-EB polymers enhance the cytosolic delivery and immunostimulatory activity of cGAMP, ${ }^{41}$ and, therefore, we assessed the relative capacity of nanoparticles assembled via FNP to load and deliver cGAMP. Polymersomes offer the potential to protect and improve the delivery of STING to the tumor site, in turn enhancing the induced immune response of cGAMP. The EE and LC of cGAMP loaded 
into $P E G_{2 k}-E_{6 k}$ and $P E G_{2 k}-E_{12 k}$ nanocarriers was largely unaffected by increasing second block molecular weight (Fig. 10A, B), similar to what was observed for these same polymers after loading with SRB (Fig. 6A). Both $P E G_{2 k}-E_{6 k}$ and $P E G_{2 k}-E_{12 k}$ STANs formulated using FNP elicited significantly enhanced activity in THP1-Dual reporter cells (Fig. 10C) compared to free cGAMP, with the $P E G_{2 k}-E_{12 k}$ enhancing activity to the greatest degree. Future in vivo analysis in murine models of cancer will be necessary to determine the relative therapeutic efficacy of STANs assembled via FNP relative to those prepared via the direct hydration method with vesicle membrane crosslinking.

\section{Conclusion}

Nanocarriers with $\mathrm{pH}$-responsive, endosome-destabilizing activity can enable the intracellular delivery of large and/or hydrophilic therapeutics that cannot freely pass through the cell membrane to engage cytosolic targets. Herein, we report that flash nanoprecipitation allows for the production of endosomolytic polymeric nanocarriers for cytosolic drug delivery with small size $(<100 \mathrm{~nm})$ and low polydispersity (PDI $<0.1)$ on the timescale of milliseconds. Additionally, all four reported PEG-EB nanocarriers were found to effectively encapsulate a variety of hydrophilic cargo and were able to disassemble and release cargo in response to a decrease in $\mathrm{pH}$ consistent with endosomal acidification, in a manner correlating with second-block molecular weight. Ultimately, these results motivate further in vitro testing of this system as well as future in vivo immunotherapy studies in murine tumor models. 


\section{References}

1. Dowdy, S. F. Overcoming cellular barriers for RNA therapeutics. Nat. Biotechnol. 35, 222-229 (2017).

2. Yin, H. et al. Non-viral vectors for gene-based therapy. Nat. Rev. Genet. 15, 541-555 (2014).

3. Pack, D. W., Hoffman, A. S., Pun, S. \& Stayton, P. S. Design and development of polymers for gene delivery. Nat. Rev. Drug Discov. 4, 581-593 (2005).

4. Varkouhi, A. K., Scholte, M., Storm, G. \& Haisma, H. J. Endosomal escape pathways for delivery of biologicals. J. Control. Release 151, 220-228 (2011).

5. Wilson, J. T. et al. Enhancement of MHC-I Antigen Presentation via Architectural Control of pH-Responsive, Endosomolytic Polymer Nanoparticles. AAPS J. 17, 358-369 (2015).

6. Wilson, J. T. et al. PH-responsive nanoparticle vaccines for dual-delivery of antigens and immunostimulatory oligonucleotides. ACS Nano 7, 3912-3925 (2013).

7. Convertine, A. J., Benoit, D. S. W., Duvall, C. L., Hoffman, A. S. \& Stayton, P. S. Development of a novel endosomolytic diblock copolymer for siRNA delivery. J. Control. Release 133, 221-229 (2009).

8. Nelson, C. E. et al. Balancing cationic and hydrophobic content of PEGylated siRNA polyplexes enhances endosome escape, stability, blood circulation time, and bioactivity in vivo. ACS Nano 7, 8870-8880 (2013).

9. Werfel, T. A. et al. Combinatorial optimization of PEG architecture and hydrophobic content improves ternary siRNA polyplex stability, pharmacokinetics, and potency in vivo. J. Control. Release 255, 12-26 (2017).

10. Peeler, D. J. et al. pH-sensitive polymer micelles provide selective and potentiated lytic capacity to venom peptides for effective intracellular delivery. Biomaterials 192, 235-244 (2019).

11. Shae, D. et al. Endosomolytic polymersomes increase the activity of cyclic dinucleotide STING agonists to enhance cancer immunotherapy. Nat. Nanotechnol. 14, 269-278 (2019).

12. Nguyen, D. C. et al. Amphiphilic Polyelectrolyte Graft Copolymers Enhance the Activity of Cyclic Dinucleotide STING Agonists. Adv. Healthc. Mater. 2001056, 1-10 (2020).

13. Shae, D. et al. Co-Delivery of Peptide Neoantigens and Stimulator of Interferon Genes (STING) Agonists Enhances Response to Cancer Vaccines. ACS Nano (2020) doi:10.1021/acsnano.0c02765.

14. Gao, P. et al. Cyclic $\left[\mathrm{G}\left(2^{\prime}, 5^{\prime}\right) \mathrm{pA}\left(3^{\prime}, 5^{\prime}\right) \mathrm{p}\right]$ is the metazoan second messenger produced by DNA-activated cyclic GMP-AMP synthase. Cell 153, 1094-1107 (2013).

15. Diner, E. J. et al. The Innate Immune DNA Sensor cGAS Produces a Noncanonical 
Cyclic Dinucleotide that Activates Human STING. Cell Rep. 3, 1355-1361 (2013).

16. Ablasser, A. et al. CGAS produces a 2'-5'-linked cyclic dinucleotide second messenger that activates STING. Nature 498, 380-384 (2013).

17. Zhang, X. et al. Cyclic GMP-AMP containing mixed Phosphodiester linkages is an endogenous high-affinity ligand for STING. Mol. Cell 51, 226-235 (2013).

18. Gao, P. et al. Structure-function analysis of STING activation by $c\left[G\left(2^{\prime}, 5^{\prime}\right) \mathrm{pA}\left(3^{\prime}, 5^{\prime}\right) \mathrm{p}\right]$ and targeting by antiviral DMXAA. Cell 154, (2013).

19. Chen, Q., Sun, L. \& Chen, Z. J. Regulation and function of the cGAS-STING pathway of cytosolic DNA sensing. Nat. Immunol. 17, 1142-1149 (2016).

20. Corrales, L., McWhirter, S. M., Dubensky, T. W. \& Gajewski, T. F. The host STING pathway at the interface of cancer and immunity. J. Clin. Invest. 126, 2404-2411 (2016).

21. Dubensky, T. W., Kanne, D. B. \& Leong, M. L. Rationale, progress and development of vaccines utilizing STING-activating cyclic dinucleotide adjuvants. Ther. Adv. Vaccines $\mathbf{1}$, 131-143 (2013).

22. Koshy, S. T., Cheung, A. S., Gu, L., Graveline, A. R. \& Mooney, D. J. Liposomal Delivery Enhances Immune Activation by STING Agonists for Cancer Immunotherapy. Adv. Biosyst. 1, 1-12 (2017).

23. Wang-Bishop, L. et al. Potent STING activation stimulates immunogenic cell death to enhance antitumor immunity in neuroblastoma. J. Immunother. Cancer 8, 1-17 (2020).

24. O'Neil, C. P., Suzuki, T., Demurtas, D., Finka, A. \& Hubbell, J. A. A novel method for the encapsulation of biomolecules into polymersomes via direct hydration. Langmuir 25, 9025-9029 (2009).

25. Johnson, B. K. \& Prud'homme, R. K. Chemical processing and micromixing in confined impinging jets. AlChE J. 49, 2264-2282 (2003).

26. Allen, S., Osorio, O., Liu, Y. G. \& Scott, E. Facile assembly and loading of theranostic polymersomes via multi-impingement flash nanoprecipitation. J. Control. Release 262, 91-103 (2017).

27. Bobbala, S., Allen, S. D. \& Scott, E. A. Flash nanoprecipitation permits versatile assembly and loading of polymeric bicontinuous cubic nanospheres. Nanoscale 10, 5078-5088 (2018).

28. Pustulka, K. M. et al. Flash nanoprecipitation: Particle structure and stability. Mol. Pharm. 10, 4367-4377 (2013).

29. Han, J. et al. A simple confined impingement jets mixer for flash nanoprecipitation. J. Pharm. Sci. 101, 4018-4023 (2012).

30. Kilchrist, K. V. et al. Gal8 Visualization of Endosome Disruption Predicts Carrier-Mediated Biologic Drug Intracellular Bioavailability. ACS Nano 13, 1136-1152 (2019). 
31. Manganiello, M. J., Cheng, C., Convertine, A. J., Bryers, J. D. \& Stayton, P. S. Diblock copolymers with tunable $\mathrm{pH}$ transitions for gene delivery. Biomaterials 33, 2301-2309 (2012).

32. Men, Y., Peng, F., Tu, Y., Van Hest, J. C. M. \& Wilson, D. A. Methods for production of uniform small-sized polymersome with rigid membrane. Polym. Chem. 7, 3977-3982 (2016).

33. Le Meins, J. F., Sandre, O. \& Lecommandoux, S. Recent trends in the tuning of polymersomes' membrane properties. Eur. Phys. J. E 34, (2011).

34. Discher, B. M. et al. Polymersomes: Tough vesicles made from diblock copolymers. Science (80-. ). 284, 1143-1146 (1999).

35. Hickey, J. W., Santos, J. L., Williford, J. M. \& Mao, H. Q. Control of polymeric nanoparticle size to improve therapeutic delivery. J. Control. Release 219, 536-547 (2015).

36. Singh, R. \& Lillard, J. W. Nanoparticle-based targeted drug delivery. Exp. Mol. Pathol. 86, 215-223 (2009).

37. Photos, P. J., Bacakova, L., Discher, B., Bates, F. S. \& Discher, D. E. Polymer vesicles in vivo: Correlations with PEG molecular weight. J. Control. Release 90, 323-334 (2003).

38. Suk, J. S., Xu, Q., Kim, N., Hanes, J. \& Ensign, L. M. PEGylation as a strategy for improving nanoparticle-based drug and gene delivery. Adv. Drug Deliv. Rev. 99, 28-51 (2016).

39. Kumar, V. et al. Shielding of lipid nanoparticles for siRNA delivery: Impact on physicochemical properties, cytokine induction, and efficacy. Mol. Ther. - Nucleic Acids 3, e210 (2014).

40. Zetasizer Nano Series User Manual. Malvern Instruments Ltd. (Malvern Instruments Ltd., 2013).

41. Wehbe, M. et al. Nanoparticle delivery improves the pharmacokinetic properties of cyclic dinucleotide STING agonists to open a therapeutic window for intravenous administration. J. Control. Release 330, 1118-1129 (2021). 


\section{Figures}

Table 1. Summary of polymer library

\begin{tabular}{|c|c|c|c|c|c|c|}
\hline $\begin{array}{c}\mathbf{2}^{\text {nd }} \text { Block } \\
\text { Name }\end{array}$ & $\begin{array}{c}\text { \% } \\
\text { Conversion }\end{array}$ & $\begin{array}{c}\text { Degree of } \\
\text { Polymerization }\end{array}$ & \% DEAMA & \% BMA & $\begin{array}{c}\mathbf{2}^{\text {nd }} \text { Block } \\
\text { MW }\end{array}$ & Total MW \\
\hline $6 \mathrm{kDa}$ & 78.3 & 39.9 & 61.9 & 38.1 & 6739.413 & 9000.79 \\
\hline $12 \mathrm{kDa}$ & 75.0 & 76.5 & 61.4 & 38.6 & 12904.57 & 15165.95 \\
\hline $15 \mathrm{kDa}$ & 72.5 & 92.0 & 62.6 & 37.4 & 15569.88 & 17831.26 \\
\hline $20 \mathrm{kDa}$ & 69.5 & 118.1 & 62.3 & 37.7 & 19962.69 & 22224.07 \\
\hline
\end{tabular}

MW - molecular weight $(\mathrm{g} / \mathrm{mol})$ 

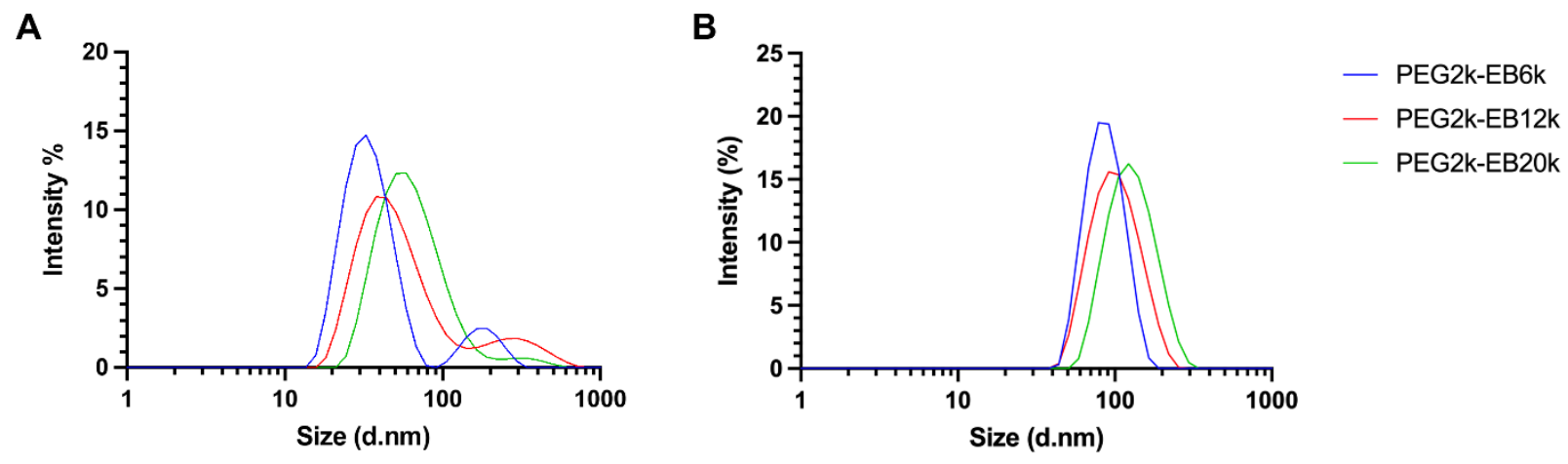

Figure 1. Effect of solvent on particle properties. Intensity-weighted size distributions for each nanocarrier fabricated with (A) ethanol and (B) tetrahydrofuran. (C) Tetrahydrofuran significantly reduced particle PDI compared to ethanol. 


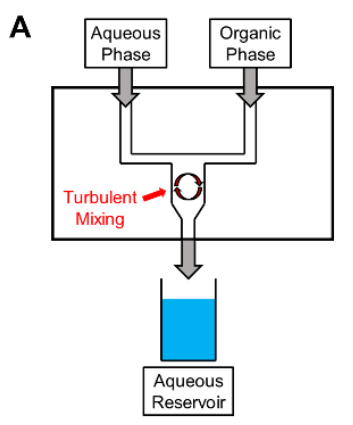

D

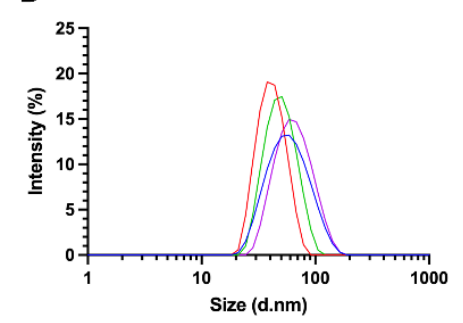

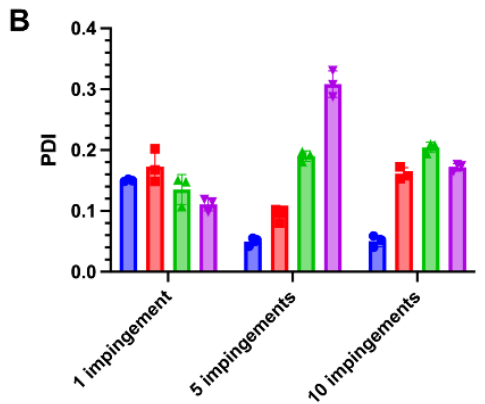

E

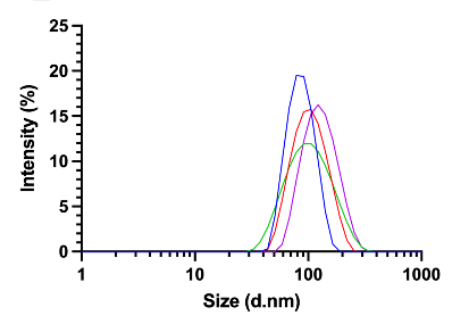

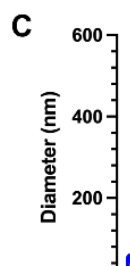

- PEG2k-EB6k

- PEG2k-EB12k

$\triangle$ PEG2k-EB15k

$\checkmark$ PEG2k-EB2OK

Figure 2. Effect of multiple impingements on particle properties. Nanocarrier (B) PDI and (C) z-average diameter were determined for one, five, and ten impingements. Intensity size distributions of all nanocarriers for (D) one, (E) five, and (F) ten impingements. Error bars denote standard deviation. 

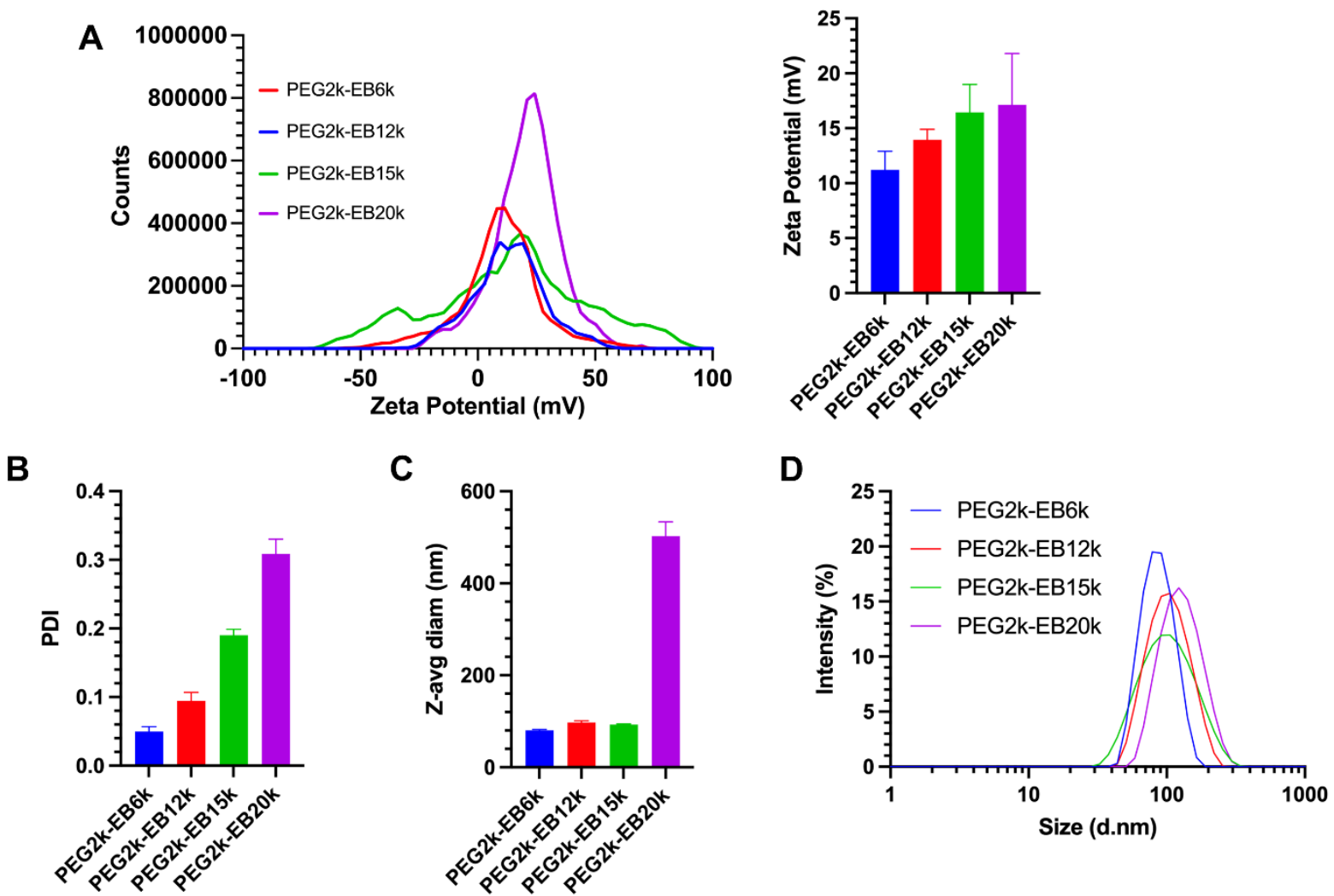

Figure 3. Particle surface charge and size. (A) Nanocarrier surface charge (zeta potential) traces and values, (B) PDI, (C) z-average diameter, and (D) intensity size distributions for five impingements. Error bars denote standard deviation. 


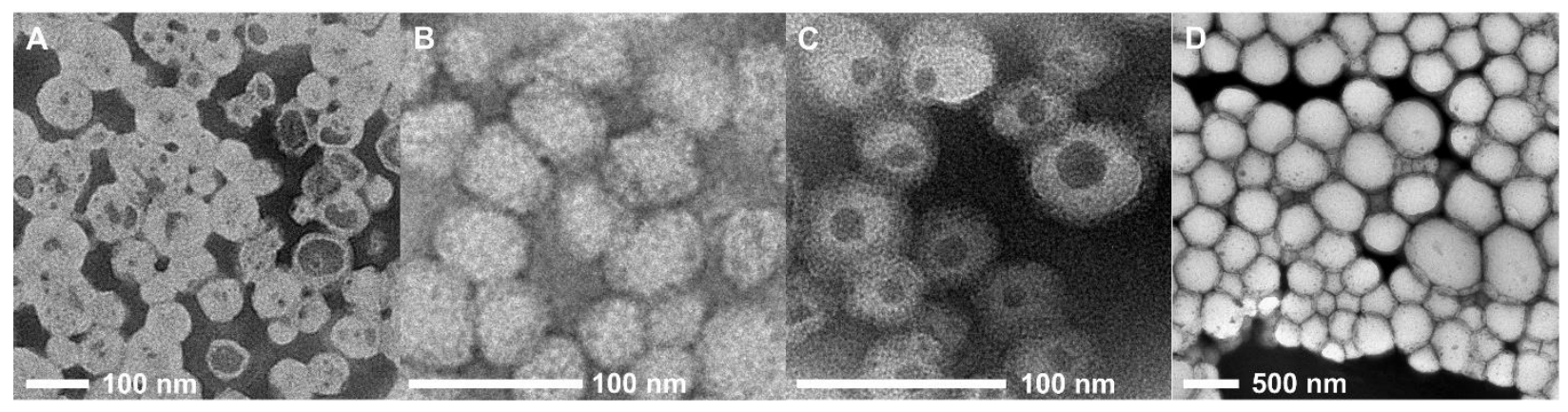

Figure 4. Morphology. Transmission electron microscopy (TEM) images of (A) $P E G_{2 k}-E_{6 k},(B)$ $\mathrm{PEG}_{2 \mathrm{k}}-\mathrm{EB}_{12 \mathrm{k},}(\mathbf{C}) \mathrm{PEG}_{2 \mathrm{k}}-\mathrm{EB}_{15 \mathrm{k},}$ and (D) $\mathrm{PEG}_{2 \mathrm{k}}-\mathrm{EB}_{20 \mathrm{k}}$ nanocarriers. 
A

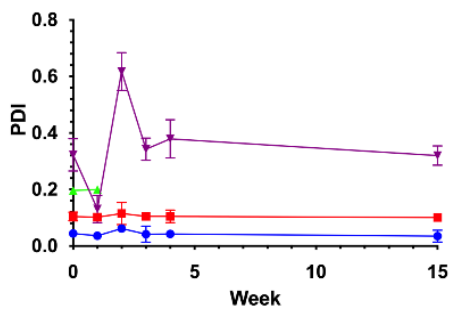

B

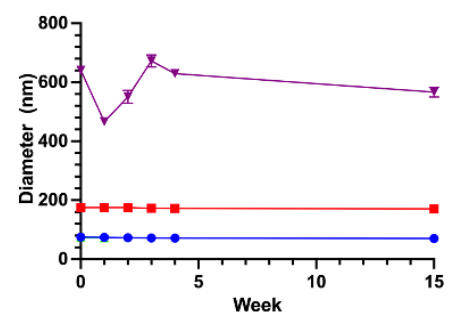

C

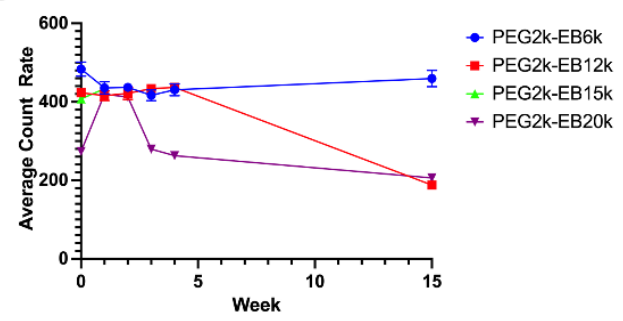

Figure 5. Colloidal stability. Nanocarrier long-term stability was assessed by measuring (A) PDI, (B) z-average diameter, and (C) count rate over four weeks. 
A
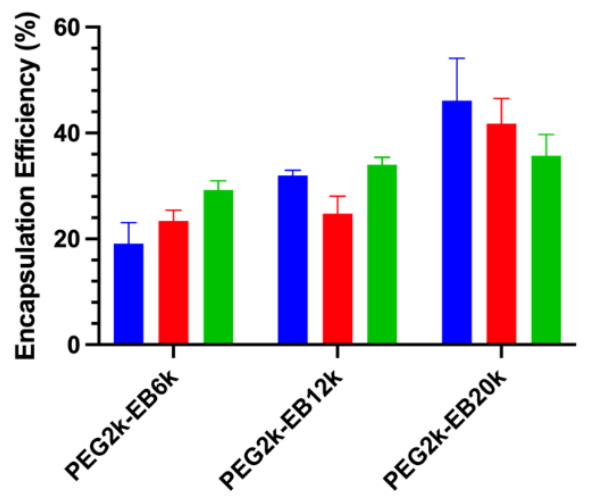

B
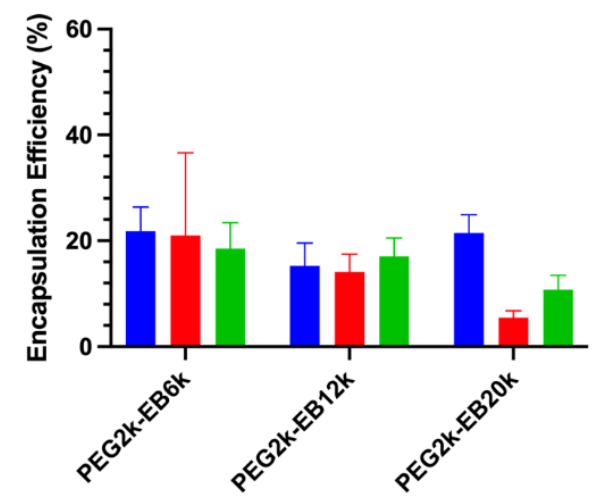

C

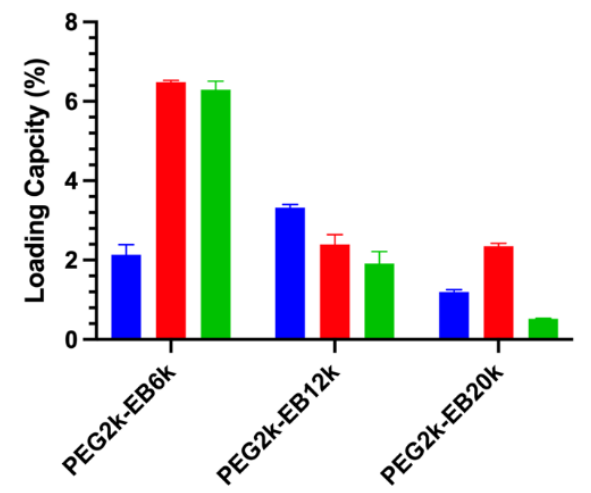

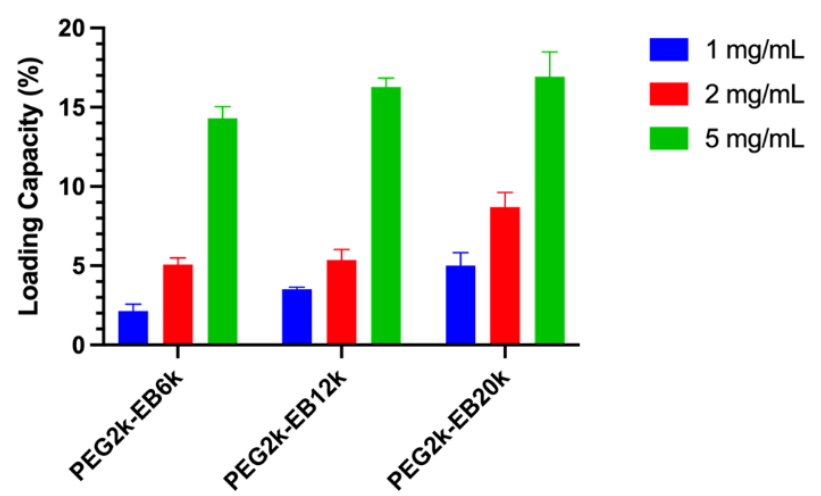
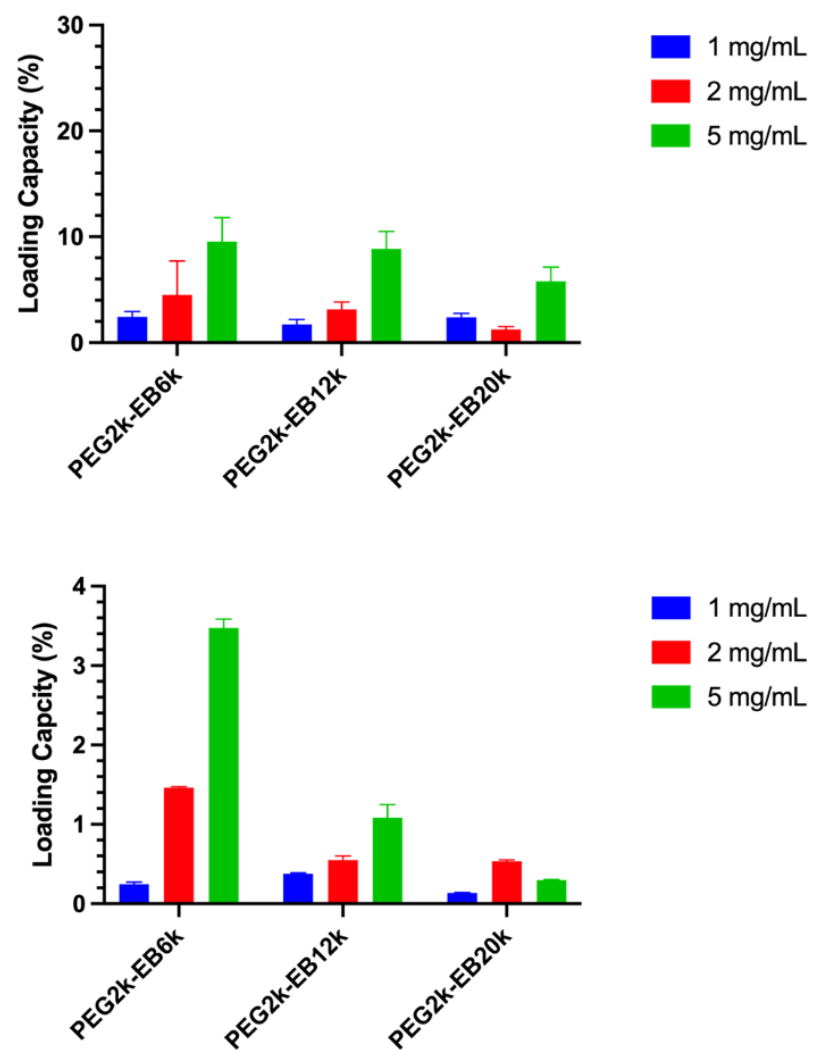

Figure 6. Loading of nanocarriers with model drugs. Encapsulation efficiency and loading capacity of (A) sulforhodamine B (SRB), (B) fluorescein isothiocyanate-Dextran (FITC-Dextran), and (C) Nile Red for each nanocarrier formulation Error bars denote standard deviation. 
A

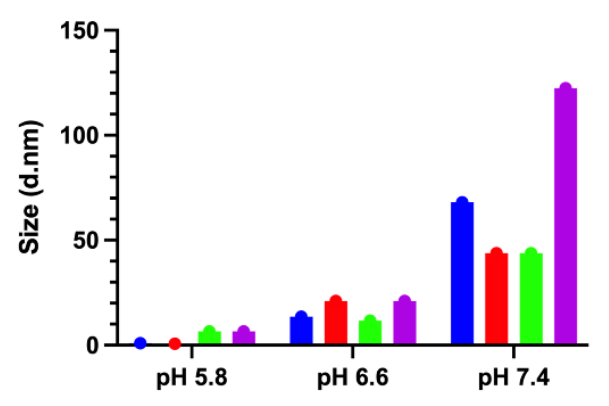

B

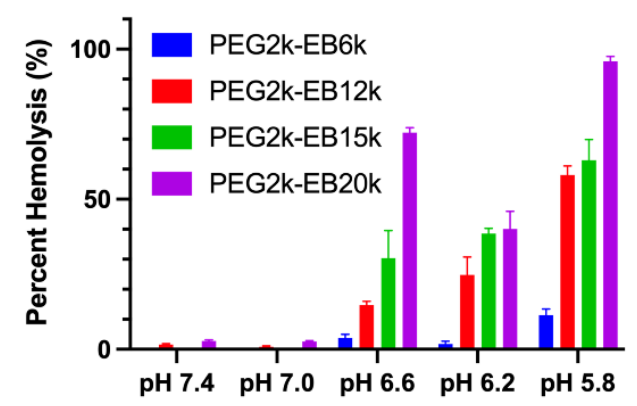

Figure 7. Nanocarrier membrane-destabilizing activity of nanocarriers. (A) Intensity size distributions indicate nanocarriers disassemble in response to a decrease in $\mathrm{pH}$. (B) Hemolysis assay indicates nanocarriers lyse erythrocytes at endosomal $\mathrm{pH}$. 

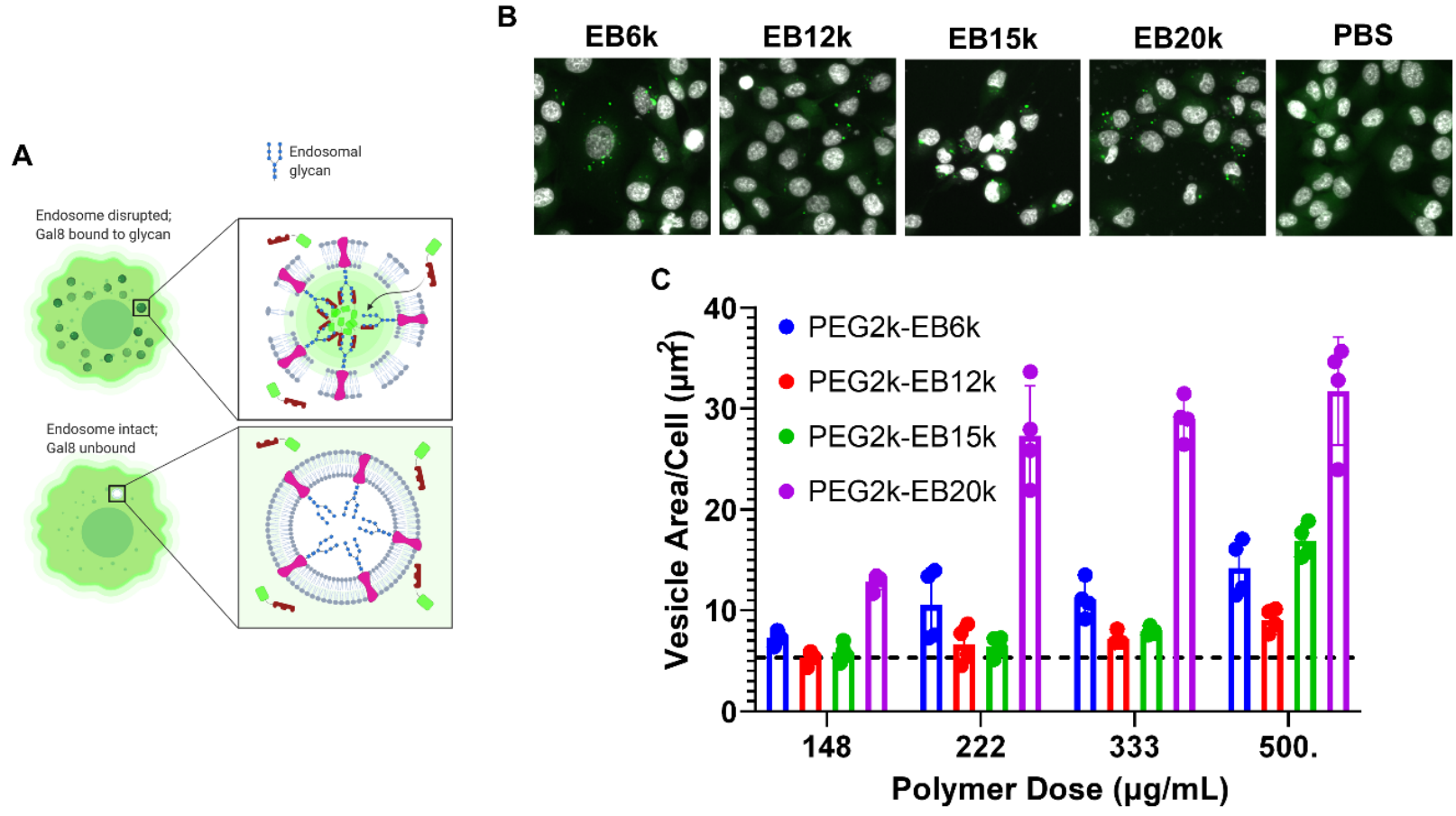

Figure 8. Gal8 recruitment/endosomal escape. (A) Schematic of Gal8-YFP assay mechanism. Reprinted with permission from ${ }^{12}$. Figure created with Biorender.com. (B) Representative images of MDA-231-MB-YFP cells treated with $500 \mu \mathrm{g} / \mathrm{mL}$ of each polymer. (C) Quantitative evaluation of endosomal puncta for each nanocarrier following a 1.5-fold dilution series. Dashed black line represents mean background puncta area observed following PBS treatment. 
A

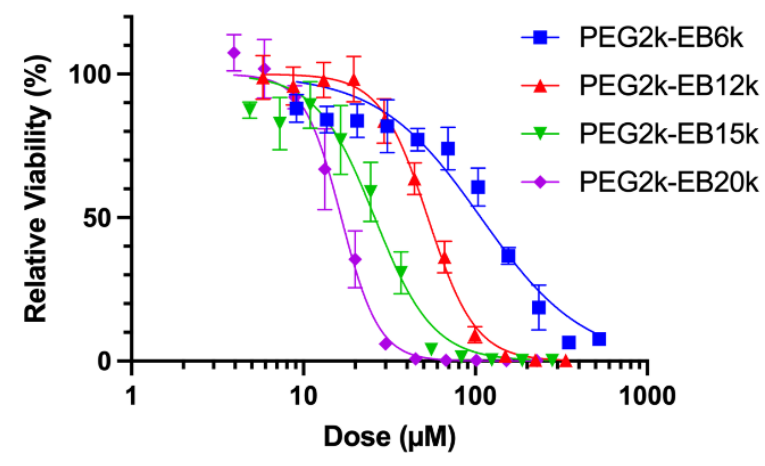

B

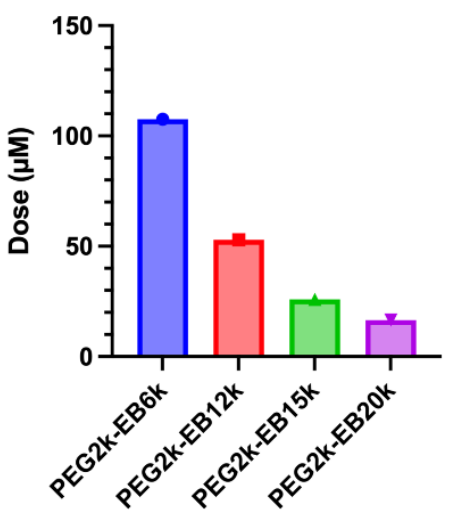

- PEG2k-EB6k

- PEG2k-EB12k

$\triangle$ PEG2k-EB15k

$\checkmark$ PEG2k-EB20k

Figure 9. Cytotoxicity. (A) Viability of A549 cells and (B) associated IC50 values after treatment with nanocarrier formulations for $24 \mathrm{~h}$. 

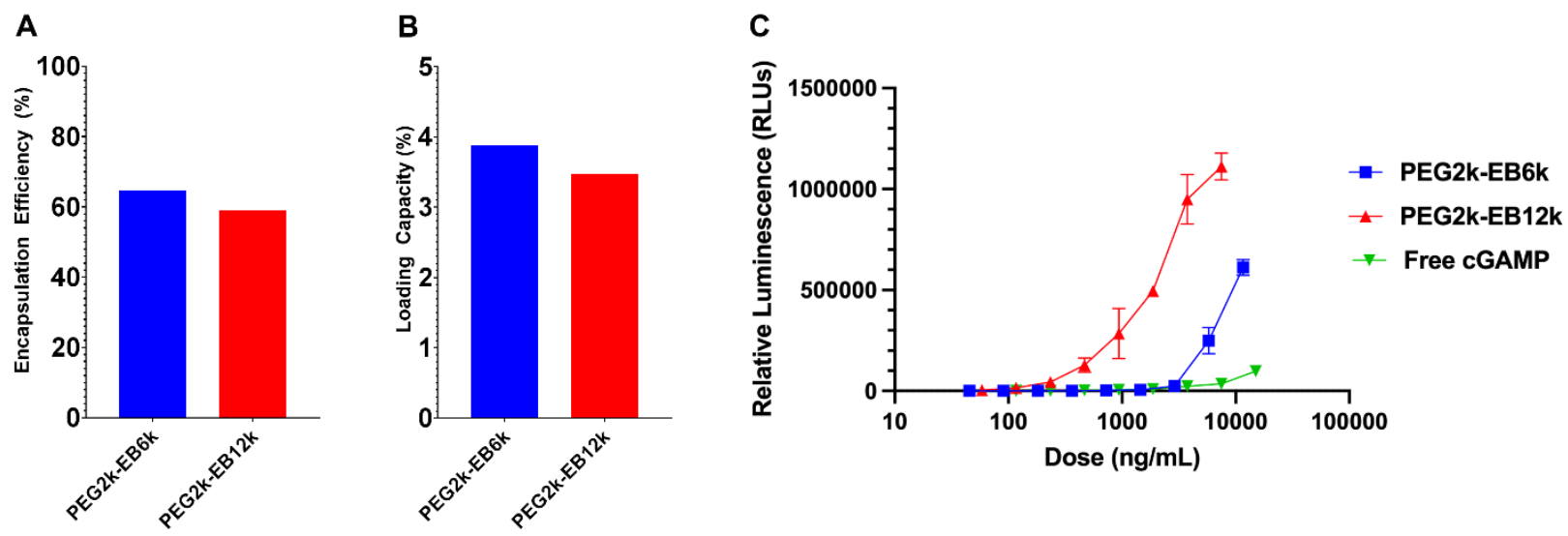

Figure 10. Nanocarrier-mediated cyclic dinucleotide delivery. (A) Encapsulation efficiency (\%) and (B) loading capacity (\%) of cGAMP loaded into $P E G_{2 k}-E_{6 k}$ and $P E G_{2 k}-E_{12 k}$ nanocarriers. (C) $P E G_{2 k}-E_{6 k}$ and $P E G_{2 k}-E_{12 k}$ nanocarriers enhanced STING activity in THP1Dual reporter cells compared to free cGAMP. 